\title{
Correlación entre la personalidad y los factores de la Teoría del Comportamiento Planeado (TCP) en adolescentes escolarizados de 11-19 años del Caribe Colombiano
}

\section{Correlation between personality and the factors of the Theory of Planned Behavior, in school enrolled adolescents from 11-19 years old in the Colombian Caribbean.}

\author{
Ana Mercedes Bello-Villanueva \\ Jorge Palacio \\ Melissa Rodríguez-Díaz \\ Grupo de investigaciones en Desarrollo Humano (GIDHUM), Departamento de Psicología, Universidad del Norte, \\ Barranquilla, Colombia. \\ Oscar Oviedo-Trespalacios \\ Grupo de investigaciones en Productividad y Competitividad, Departamento de Ingeniería Industrial, Universidad del \\ Norte, Barranquilla, Colombia. \\ Queensland University of Technology (QUT), Centre for Accident Research and Road Safety - Queensland (CARRS-Q), \\ Australia
}

(Rec: 31 enero 2013 / Acept: 20 octubre 2015)

\begin{abstract}
Resumen
La creciente tasa de embarazos en adolescentes y la alta incidencia de las infecciones de transmisión sexual (entre ellas el VIH/SIDA), son temáticas en torno a la salud y en especial la salud sexual y reproductiva, que han recibido gran atención por parte de investigadores y de la opinión pública en general. Recientemente, se ha encontrado que los adolescentes llevan a cabo con facilidad comportamientos de riesgo para su salud sexual y reproductiva y aquellos que no han presentado dichos comportamientos también muestran altos niveles de intención para llevarlos a cabo. Se ha hipotetizado que además de variables cognitivas como las actitudes, normas subjetivas, control conductual percibido y la intención, la personalidad de los jóvenes es un aspecto que juega un papel importante en su salud sexual y reproductiva. Se encontraron correlaciones significativas entre las variables de la TCP y las características de personalidad y los resultados sugieren que la direccionalidad de estas correlaciones se encuentra asociada con el tipo específico de comportamiento o situación que se está evaluando. Palabra claves: personalidad, teoría del comportamiento planeado, adolescentes, sexualidad reproductiva.
\end{abstract}

\begin{abstract}
The increasing rate of pregnancies in teenagers and the high incident of the infections of sexual transmission (HIV/ AIDS, for example), these are health related issues (and especially the sexual and reproductive health), which have received great attention on the part of investigators and of the public opinion in general. Recently, there has been evidenced that teenagers carry out very easily risk sexual behaviors, and those who have not presented the above mentioned behaviors also show high levels of intention to carry out them. There is the hypothesis that besides cognitive variables such as attitudes, subjective norms, perceived behavioral control and intention, the personality of the young persons is an aspect that plays an important paper in their sexual and reproductive health. Significant correlations were found between the variales of the TPB and the personality traits; the results suggest that the direction of these correlations is associated with the specific type of behavior or situation that is assessed. Keywords: personality, theory of planned behavior, adolescents, reproductive sexuality.
\end{abstract}

\footnotetext{
Correspondencia: Ana Mercedes Bello, Universidad del Norte, A.A. 1569-15820 Barranquilla - Atlántico, Colombia. Email: ambellov@yahoo.com bellom@uninorte.edu.co

Agradecimientos: Esta investigación ha sido financiada por Colciencias como parte del proyecto código No 121549326168, denominado: "validación de estrategias pedagógicas y comunicacionales mediante un modelo cognitivo social y epidemiológico para la prevención de comportamientos sexuales de riesgo, en adolescentes escolarizados entre 11-16 años de dos ciudades del Caribe colombiano" junto con el apoyo de la DIP, Universidad del Norte, Barranquilla-Colombia.
} 


\section{Introducción}

Las investigaciones basadas en las actitudes, las creencias y en la intención de actuar o no, reflejan correlaciones significativas y empíricamente relevantes en la adopción de comportamientos sexuales protectores para el desarrollo de programas en prevención de embarazos no deseados, ITS (Infecciones de transmisión sexual) y el Virus de Inmundodeficiencia Humana (VIH/SIDA) en adolescentes (Baele, Dusseldorp y Maes, 2001; Flores, Tschann y Marin, 2002; Vargas-Trujillo, Barrera, Burgos y Daza, 2006).

La sexualidad adolescente se ha constituido en una temática de interés general y científico y ha sido estudiada desde disciplinas y modelos dentro de cada disciplina, que buscan el cambio de comportamientos de riesgo hacia comportamientos saludables. En la psicología, estas conductas se han estudiado desde enfoques teóricos como el Modelo de Creencias en Salud, el Modelo Transteórico, la Teoría de la Acción Razonada (TRA), la Teoría del Comportamiento Planeado (TCP), entre otros. Igualmente, se ha planteado la necesidad de tener en cuenta características estables y duraderas en los adolescentes como es la personalidad, los cuales -en conjunto- han demostrado capacidad de predicción de las conductas de riesgo de los adolescentes.

De acuerdo con la Encuesta Nacional de Demografía y Salud (ENDS) en Colombia del año 2010, amplios porcentajes de la población adolescente ha llevado a cabo comportamientos de riesgo como inicio temprano de las relaciones sexuales $(60.3 \%)$, no utilización del condón (49\%), promiscuidad (30.2\%) y promiscuidad sin usar ningún método de protección (49.9\%) (Profamilia, 2010), estadística que se acompaña de una alta incidencia de embarazos adolescentes: 1 de cada 5 adolescentes entre los 15 y 19 años, ha estado alguna vez embarazada (Profamilia, 2010). Adicionalmente, en 2012 en Colombia existían cerca de 96 mil personas infectadas con el VIH y para el $2014-2015$ pueden estar entre 120 y 144 mil personas (Ministerio de Salud y Protección Social, 2013).

El inicio temprano de las relaciones sexuales (antes de los 15 años) (Departamento Nacional de Planeación, 2012; Ntaganira, Hass, Hosner, Brown y Mock, 2012) y el uso deficiente de los métodos de planificación familiar se encuentran asociados a los embarazos no deseados en adolescentes y las ITS; sin embargo, los factores que han demostrado tener mayor impacto sobre el comportamiento de los jóvenes alrededor de su salud sexual y reproductiva, son las características personales de los sujetos tales como las actitudes, las normas subjetivas, la asertividad, autoeficacia, toma de decisiones, intención y la personalidad (Carmack y Lewis-Moss, 2009; Charnigo et al., 2012; Fishbein y Ajzen, 1975; Fontanilla, Bello y Palacio, 2011; Martín, Martínez y Rojas, 2011; Papalia, Wendkos y Duskin, 2009).

De acuerdo con la Teoría de Acción Razonada (Fishbein y Ajzen, 1975), las conductas están determinadas por las actitudes, las normas subjetivas y el control percibido; posteriormente Ajzen (2001), afirma que el antecedente inmediato de la conducta no es la actitud como explica la TRA, sino la intención de ejecutarla. Es este el modelo de la Teoría del Comportamiento Planeado, cuya utilidad para el estudio y la predicción de los comportamientos sexuales de riesgo de los adolescentes ha sido demostrada en varios contextos (Carmack y Lewis-Moss, 2009; Jemmott et al. 2007; Martín, et al., 2011).

Igualmente, se ha demostrado el rol que juegan las características de personalidad en la adopción de comportamientos protectores y de riesgo en salud sexual y reproductiva (DiClemente et al., 2008; Llewellyn, 2008; Ntaganira, et al., 2012). Eysenck (1970a, 1970b), afirma que la personalidad comprende dos dimensiones: Extraversión - Introversión y Neuroticismo (emocionabilidad o inestabilidad) - Estabilidad. Existe una tercera dimensión con funcionalidad independiente llamada Psicoticismo.

En la dimensión neuroticismo-estabilidad, el neuroticismo hace referencia a una persona ansiosa, con altos niveles de preocupación y frecuentes cambios de humor en los que se incluyen estados depresivos; suele tener quejas somáticas y dificultades para el sueño, es muy emotivo y reacciona con una mayor valencia que las demás personas ante todo tipo de estímulos y le cuesta trabajo estabilizar sus emociones después de esta respuesta (Eysenck y Eysenck, 2007). En la dimensión extraversión-introversión, el sujeto extravertido es sociable, con muchos amigos y atracción hacia las fiestas, conversador y con poca tendencia a permanecer solo así sea estudiando o leyendo. Le gustan las emociones fuertes, es arriesgado y generalmente impulsivo; le gustan las bromas y es muy astuto, despreocupado, optimista, con tendencia a la agresión e irritable. Le cuesta trabajo mantener control sobre sus propias emociones y regularmente no es una persona en quien confiar (Eysenck y Eysenck, 2007). La dimensión de psicoticismo o dureza hace referencia a sujetos solitarios y despreocupados de los demás. Puede resultar cruel, inhumano, insensible y poco empático, hostil y agresivo incluso con personas cercanas y seres queridos (Eysenck y Eysenck, 2007). Al igual que en el caso de las variables de la TCP, se ha demostrado el rol que juegan las características de personalidad en la adopción de 
comportamientos protectores y de riesgo en salud sexual y reproductiva (DiClemente, et al., 2008; Llewellyn., 2008; Ntaganira, et al., 2012).

El estudio de la relación entre las variables de la TCP y las características de personalidad, se remonta a los años 70, gracias a Eysenck (1970), quien elaboró un cuestionario para medir personalidad y actitudes hacia el sexo, el cual determinó que sí existe correlación entre estas variables, en población normal e incluso en población que se ha visto involucrada en conductas criminales (Eysenck, 1973). Adicionalmente, diversos estudios han evidenciado la relación que existe entre los factores de personalidad y las conductas de riesgo: se ha demostrado que la conducta antisocial, la extraversión y el neuroticismo predicen el consumo de tabaco y sólo la conducta antisocial y la extraversión resultaron predictores del consumo de alcohol (Inglés et al., 2006). Otros estudios han demostrado que los comportamientos de riesgo se relacionan con la búsqueda de sensaciones y la impulsividad: el consumo de alcohol se encuentra relacionado con comportamientos de riesgo para el contagio de VIH (Kalichman, Simbayi, Jooste, Cain y Cherry, 2006) e igualmente, tanto el consumo de alcohol como los comportamientos de riesgo para contraer VIH, se encuentran relacionados con la búsqueda de sensaciones (Charnigo, et al., 2012; Kalichman, Cain, Zweben y Swain, 2003; Kalichman, Simbayi, Jooste, Vermaak y Cain, 2008) y con los niveles de impulsividad, agresión y sociabilidad (Zuckerman y Kuhlman, 2000). Sin embargo, aún no se conoce con certeza la relación que existe entre las variables de personalidad y otras características psicológicas en el marco del estudio de la sexualidad humana y en especial la sexualidad adolescente, la cual tuvo inicio en 1970 con los estudios de Eysenck, pero que no han tenido eco en estudios más recientes que permitan soportar o reformular sus hallazgos.

De acuerdo a lo anterior, esta investigación se realizó para probar la hipótesis sobre la existencia de una correlación entre las características de personalidad y las variables de la TCP, en el marco de las conductas sexuales de la población adolescente en la región norte de Colombia. Los resultados se derivan de la línea base de un macro proyecto de investigación financiado por Colciencias y la Universidad del Norte, titulado "Validación de estrategias pedagógicas y comunicacionales mediante un modelo cognitivo social y epidemiológico para la prevención de comportamientos sexuales de riesgo, en adolescentes escolarizados entre 11-16 años de dos ciudades del Caribe colombiano".

\section{Método}

\section{Participantes}

La muestra estuvo conformada por 1.581 estudiantes escolarizados - 811 hombres (51.29\%) y 770 mujeres (48.71\%) - seleccionados mediante un muestreo intencional por criterio, cuyas edades se encontraban entre los 11 y 19 años $(M=14.2 ; D E=2.0)$, residentes de cinco ciudades de la región Caribe Colombiana (Ver tabla 1).

Tabla 1. Datos Sociodemográficos de la muestra

\begin{tabular}{lcc}
\hline Variables & & $\mathrm{N}$ \\
\hline \multirow{2}{*}{ Género } & Masculino & 811 \\
& Femenino & 770 \\
Grado de escolaridad & $6^{\circ}-7^{\circ}$ & 588 \\
& $8^{\mathrm{o}}-9^{\circ}$ & 602 \\
& $10^{\circ}-11^{\circ}$ & 580 \\
\hline
\end{tabular}

En algunos análisis, las sumas de los totales por cada variable no coinciden con la muestra total de 1.581 estudiantes, por efecto de los datos perdidos y porque se toman sólo los porcentajes válidos. Un $14.7 \%$ de las adolescentes y $32.6 \%$ de los adolescentes, reportaron experiencia sexual. Adicionalmente, 788 adolescentes (79.8\%) reportaron estar involucrados en relaciones afectivas al momento de diligenciar la encuesta.

\section{Instrumentos}

SR-F (Cuestionario de Sexualidad Reproductiva Forma Femenina) y SR-M (Cuestionario de Sexualidad Reproductiva Forma Masculina) (Bello y Palacio, 2012): son cuestionarios de autorreporte que evalúan actitudes, normas subjetivas, control conductual percibido, intención, asertividad, toma de decisiones (cada una de estas variables se organizaron en subescalas) y comportamientos (que arrojan un índice de riesgo) en relación con la sexualidad adolescente, indagando adicionalmente aspectos sociodemográficos y de la dinámica familiar según la percepción del adolescente. Los Instrumentos fueron validados con altos niveles de confiabilidad que fluctúan entre .74 a .98 para todas las escalas (Bello-Villanueva, Palacio, Rodríguez-Díaz y Oviedo-Trespalacios, 2013; Bello-Villanueva et al., 2014; Bello-Villanueva et al., 2016). Alta puntuación en la escala, indica altos niveles de cada variable (favorabilidad de las actitudes, percepciones de la norma subjetiva y del control conductual, alto nivel de intención, alto nivel de asertividad y de capacidad de toma de decisiones, así como altos 
niveles de riesgo para el caso del índice del comportamiento de riesgo sexual).

EPQ (Eysenck Personality Questionnaire-Junior EPQ-J and Adult EPQ-A), Cuestionario de Personalidad para Niños (EPQ-J) y Adultos (EPQ-A): Test de aplicación individual y colectiva en español a sujetos de 8 a 15 años (EPQ-J) y de 16 años en adelante (EPQ-A). El tiempo de aplicación oscila entre los 20 y 30 minutos y tiene como finalidad evaluar las tres dimensiones básicas de la personalidad: neuroticismo, extraversión y psicoticisimo o dureza, así como una escala de sinceridad, cuyo referente operativo se encuentra en los percentiles en los cuales se traducen las puntuaciones brutas obtenidas en cada una de ellas (Eysenck y Eysenck, 2007). La versión para jóvenes cuenta con 81 reactivos y la versión para adultos tiene 92 , los cuales consisten en preguntas sencillas cuyas opciones de respuesta son dicotómicas - Sí y No.

\section{Procedimiento}

Inicialmente, se llevó a cabo el muestreo de acuerdo con la descripción anterior y una vez se seleccionaron los colegios, se procedió a la firma de las cartas de compromiso por parte de los colegios que accedieron a participar; posteriormente, se enviaron circulares informando sobre el proyecto y en los casos en que los colegios así lo requirieron, se programaron reuniones con los padres de familia, con el fin de dar a conocer las principales características del estudio así como los objetivos y alcances del mismo, con el fin de que los padres tomaran la decisión sobre la autorización para que sus hijos hicieran parte del presente estudio.
Los estudiantes que firmaron el consentimiento informado y cuyos padres también lo hicieron, fueron quienes participaron en el diligenciamiento de los instrumentos aplicados. Dicha aplicación fue realizada por el equipo de trabajo de la investigación, conformado por 2 psicólogas, 1 enfermera y 2 comunicadoras sociales, así como grupos de apoyo que fue previamente entrenado y capacitado para brindar orientación a los jóvenes que así lo solicitaran durante la sesión de evaluación. Para cada grupo de estudiantes, se realizó una única sesión de evaluación con descansos establecidos para controlar el efecto de la fatiga. Posteriormente, se llevó a cabo la tabulación de los datos recopilados, la depuración de las bases de datos con el fin de verificar la utilidad de los datos y posteriormente se dio inicio al proceso de análisis estadístico con el Paquete Estadístico SPSS Versión 19.0. Se realizaron análisis descriptivos así como análisis de correlación mediante el Estadígrafo de Correlación r de Pearson (Sierra, Oviedo-Trespalacios, Candelo y Soto, 2015). Los resultados fueron analizados y seguidamente se llevó a cabo la discusión de los mismos.

\section{Resultados}

A nivel general no se encontraron resultados consistentes en las correlaciones entre las dos versiones del EPQ y la actitud; las correlaciones se encuentran en la Tabla 2, en la cual se sombrean en gris las correlaciones significativas.

Los resultados de correlación entre personalidad y normas subjetivas serán presentados en tres grupos, teniendo en cuenta los tres factores que componen la norma subjetiva.

Tabla 2. Índices de correlación entre las dimensiones de personalidad y las actitudes

\begin{tabular}{llcc}
\hline & & ACT1 & ACT2 \\
\hline \multirow{2}{*}{ Cuestionario de Personalidad de Jóvenes } & Neuroticismo & .058 & .104 \\
& Extraversión & $.212^{* *}$ & $.256^{* *}$ \\
& Psicoticismo & -.138 & $-.215^{* *}$ \\
& Sinceridad & $.242^{* *}$ & .051 \\
& Neuroticismo & $-.213^{* *}$ & .049 \\
Cuestionario de Personalidad de Adultos & Extraversión & .100 & -.046 \\
& Psicoticismo & $-.154^{*}$ & -.043 \\
\hline
\end{tabular}

ACT1: Actitud hacia la protección

ACT2: Actitud hacia la abstinencia o el retardo

Nota. $* \mathrm{p}<.05 ; * * \mathrm{p}<.01$. 
Tabla 3. Índices de correlación entre las dimensiones de personalidad y la percepción de apoyo y aprobación de los grupos de referencia.

\begin{tabular}{ccccccc}
\hline \multirow{6}{*}{ Jóvenes } & & NS1 & NS2 & NS3 & NS4 & NS5 \\
& Neuroticismo & .071 & .071 & $.150^{*}$ & .093 & .057 \\
& Extraversión & $.318^{* *}$ & $.228^{* *}$ & $.284^{* *}$ & $.241^{* *}$ & $.242^{* *}$ \\
& Psicoticismo & $-.190^{*}$ & $-.206^{* *}$ & -.146 & -.090 & -.100 \\
\multirow{3}{*}{ Adultos } & Sinceridad & $.166^{*}$ & .115 & .135 & .074 & $-.153^{*}$ \\
& Neuroticismo & $-.241^{* *}$ & $-.223^{* *}$ & $.153^{*}$ & .092 & $.152^{*}$ \\
& Extraversión & $.142^{*}$ & .114 & .067 & -.044 & -.017 \\
& Psicoticismo & -.101 & $-.153^{*}$ & -.075 & -.094 & .089 \\
& Sinceridad & -.068 & $-.143^{*}$ & -.003 & -.013 & -.014 \\
\hline
\end{tabular}

NS1: Aprobación y apoyo de personas adultas protección (AE, AO)

NS2: Aprobación y apoyo de amigos protección (AE, AO)

NS3: Aprobación y apoyo de padres en abstinencia/retardo

NS4: Aprobación y apoyo de profesores en abstinencia/retardo

NS5: Aprobación y apoyo de amigos abstinencia/retardo

Nota. $* \mathrm{p}<.05 ; * * \mathrm{p}<.01$.

Tabla 4. Índices de correlación entre las dimensiones de personalidad y la disposición a complacer a los grupos de referencia

\begin{tabular}{llll}
\hline & & NS6 & NS7 \\
\hline \multirow{3}{*}{ Jóvenes } & Neuroticismo & .086 & .031 \\
& Extraversión & $.230^{* *}$ & $.246^{* *}$ \\
& Psicoticismo & $-.220^{* *}$ & $-.235^{* *}$ \\
& Sinceridad & .074 & -.040 \\
\hline \multirow{3}{*}{ Adultos } & Neuroticismo & $-.147^{*}$ & .054 \\
& Extraversión & .089 & -.030 \\
& Psicoticismo & $-.211^{* *}$ & -.058 \\
& Sinceridad & -.104 & -.117
\end{tabular}

NS6: Dispuesto a complacer padres, profesores y amigos en protección (AE, AO)

NS7: Dispuesto a complacer padres, profesores y amigos en abstinencia/retardo

Nota. $* \mathrm{p}<.05 ; * * \mathrm{p}<.01$.

Para el primer factor (Percepción de apoyo y aprobación de los grupos de referencia), se observa que todas las escalas de personalidad para ambas versiones tienen correlaciones significativas con al menos una de las sub escalas de norma subjetiva referidas a la percepción de apoyo por parte de los grupos de referencia para el uso de los métodos de protección y la práctica de la abstinencia/retardo (Ver tabla 3).

En lo referente a la norma subjetiva, específicamente qué tan dispuesto se siente el joven a complacer la norma de los grupos de referencia, los resultados se encuentran en la Tabla 4.

En las dos últimas sub escalas de la norma subjetiva (Tabla 5), se encontró que la escala de neuroticismo no correlacionó con ninguna de ellas, en ninguno de los grupos etarios. La extraversión y el psicoticismo correlacionaron significativamente para ambas sub escalas de normas subjetivas pero únicamente en el grupo de los adolescentes más jóvenes y ambas escalas de personalidad tienen direcciones diferentes en sus correlaciones.

Los resultados que conciernen a las correlaciones de la personalidad con las sub escalas de control conductual percibido en el grupo de adolescentes sin experiencia sexual, resultan en algún grado similares a la tendencia observada en las variables anteriores. Por ejemplo, se encontró evidencia de una relación significativa y positiva entre la extraversión en los jóvenes de 11 a 15 años y todas las escalas de control 
Tabla 5. Índices de correlación entre las dimensiones de personalidad y la importancia a los grupos de referencia.

\begin{tabular}{llcc}
\hline & & NS8 & NS9 \\
\hline \multirow{4}{*}{ Jóvenes } & Neuroticismo & .005 & .045 \\
& Extraversión & $.218^{* *}$ & $.254^{* *}$ \\
& Psicoticismo & $-.226^{* *}$ & $-.225^{* *}$ \\
& Sinceridad & -.093 & -.047 \\
\hline \multirow{3}{*}{ Adultos } & Neuroticismo & .103 & -.032 \\
& Extraversión & .059 & -.063 \\
& Psicoticismo & -.022 & -.133 \\
& Sinceridad & $-.209^{* *}$ & $-.263^{* *}$ \\
\hline
\end{tabular}

NS8: Importancia de padres, profesores y amigos en abstinencia/retardo NS9: Importancia de padres, profesores y amigos en protección Nota. $* \mathrm{p}<.05 ; * * \mathrm{p}<.01$.

Tabla 6. Índices de correlación entre las dimensiones de personalidad y el control conductual percibido de los jóvenes sin experiencia

\begin{tabular}{lllllll}
\hline & & AUS1 & AUS2 & AUS3 & AUS4 & AUS5 \\
\hline \multirow{6}{*}{ Jóvenes } & Neuroticismo & .090 & $.165^{*}$ & .118 & .124 & .085 \\
& Extraversión & $.265^{* *}$ & $.291^{* *}$ & $.264^{* *}$ & $.252^{* *}$ & .131 \\
& Psicoticismo & $-.213^{* *}$ & $-.237^{* *}$ & -.091 & $-.184^{*}$ & $-.170^{*}$ \\
& Sinceridad & .119 & .149 & -.048 & .023 & -.022 \\
\hline \multirow{3}{*}{ Adultos } & Neuroticismo & $-.222^{* *}$ & $-.184^{* *}$ & $-.182^{*}$ & -.049 & -.102 \\
& Extraversión & -.121 & .082 & -.059 & .044 & -.120 \\
& Psicoticismo & $-.170^{*}$ & $-.172^{*}$ & .004 & -.091 & -.063 \\
& Sinceridad & $-.145^{*}$ & -.042 & -.076 & -.005 & $-.218^{* *}$ \\
\hline
\end{tabular}

AUS1: Control General

AUS2: Confio Protección

AUS3: Confio Abstinencia/Retardo

AUS4: Seguro poder Protección

AUS5: Seguro poder Abstinencia/Retardo

Nota. $* \mathrm{p}<.05 ; * * \mathrm{p}<.01$.

conductual percibido excepto aquella relacionada con el nivel de seguridad que el joven percibe para practicar la abstinencia o retardo de las relaciones sexuales.

Igualmente, se puede observar que en los jóvenes de 16 a 19 años los altos niveles de Neuroticismo se encuentra relacionados inversamente con la confianza que perciben en sí mismos a nivel general y específico (Ver Tabla 6). Es decir que los jóvenes con mayor nivel de Neuroticismo tienden a presentar menos confianza en sí mismos en estos aspectos. En el grupo de los jóvenes que reportaron experiencia sexual, se encuentran las mismas correlaciones significativas con igual dirección.

Una de las variables de la TCP que más atención ha recibido en relación con la personalidad y los comportamientos saludables, es la intención de llevar a cabo comportamientos (Ajzen y Fishbein, 2005). En jóvenes la intención de riesgo muestra una asociación negativa y significativa con la escala de Neuroticismo, mientras que la intención de protección muestra una correlación significativa y positiva con las escalas de extraversión y sinceridad. Así mismo, en adultos la escala de Neuroticismo muestra una asociación negativa y significativa con la intención de riesgo, mientras que la escala de sinceridad se asoció significativamente con signo contrario. La escala de Neuroticismo y Psicoticismo correlacionaron de forma negativa con la escala de intención de protección.

El estudio de la personalidad en relación con la adopción de comportamientos de riesgo se ha venido adelantando hace varios años (Hoyle, Fejfar y Miller, 2000), evidenciando relación entre estos constructos (Eysenck, 1972). En la tabla 9 se encuentran los resultados en este aspecto. 
Tabla 7. Índices de correlación entre las dimensiones de personalidad y el control conductual percibido de los jóvenes con experiencia

\begin{tabular}{llll}
\hline & & AUC1 & AUC2 \\
\hline \multirow{4}{*}{ Jóvenes } & Neuroticismo & .075 & -.001 \\
& Extraversión & $.217^{* *}$ & $.224^{* *}$ \\
& Psicoticismo & $-.182^{*}$ & -.065 \\
& Sinceridad & .099 & -.084 \\
\hline \multirow{4}{*}{ Adultos } & Neuroticismo & $-.154^{*}$ & .063 \\
& Extraversión & .037 & -.021 \\
& Psicoticismo & $-.186^{*}$ & -.061 \\
& Sinceridad & $-.184^{*}$ & $-.156^{*}$ \\
\hline
\end{tabular}

AUC1: Confio y seguro

AUC2: Control/Dependencia Pareja

Nota. $* \mathrm{p}<.05 ; * * \mathrm{p}<.01$.

Tabla 8. Índices de correlación entre las dimensiones de personalidad y la intención

\begin{tabular}{llll}
\hline & & IR1 & IP2 \\
\hline \multirow{4}{*}{ Jóvenes } & Neuroticismo & $-.203^{* *}$ & .090 \\
& Extraversión & .049 & $.238^{* *}$ \\
& Psicoticismo & .044 & -.136 \\
& Sinceridad & .049 & $.171^{*}$ \\
\hline \multirow{3}{*}{ Adultos } & Neuroticismo & $-.171^{*}$ & $-.170^{*}$ \\
& Extraversión & .033 & .006 \\
& Psicoticismo & .075 & $-.225^{* *}$ \\
& Sinceridad & $.146^{*}$ & -.100 \\
\hline
\end{tabular}

IR1: Intención de Riesgo

IR2: Intención de Protección

Nota. $* \mathrm{p}<.05 ; * * \mathrm{p}<.01$.

Tabla 9. Índices de correlación entre las dimensiones de personalidad y el índice de riesgo.

\begin{tabular}{clc}
\hline & & SUMRISK \\
\hline \multirow{2}{*}{ Jóvenes } & Neuroticismo & $-.167^{*}$ \\
& Psicoticismo & .021 \\
& Sinceridad & $.175^{*}$ \\
& Neuroticismo & $.266^{* *}$ \\
\hline \multirow{2}{*}{ Adultos } & Extraversión & -.074 \\
& Psicoticismo & .063 \\
& Sinceridad & .013 \\
& & $.212^{* *}$
\end{tabular}

SUMRISK: Índice de comportamientos de riesgo

Nota. $* \mathrm{p}<.05 ; * * \mathrm{p}<.01$. 


\section{Discusión y Conclusiones}

El objetivo de esta investigación fue determinar si existen correlaciones entre las características de personalidad (Neuroticismo, Extraversión, Psicoticismo y Sinceridad) y las variables de la Teoría del Comportamiento Planeado (TCP: Actitudes, normas subjetivas, control conductual percibido, intención y comportamiento de riesgo sexual), en adolescentes de 11-19 años del Caribe Colombiano. Los resultados de correlación encontrados en esta investigación resultan, en su mayoría, novedosos, ya que las investigaciones realizadas previamente sobre el tema no abordaron otras variables de la TCP, sino exclusivamente el comportamiento y en algunos casos aislados, la intención.

Teniendo en cuenta las características expuestas por Eysenck y Eysenck sobre los sujetos que puntúan alto y bajo en la escala $\mathrm{N}$, los resultados sugieren que aquellos jóvenes que tienden a ser despreocupados consideran que sus grupos de referencia apoyan el uso de los métodos de protección (y por lógica aprueban las relaciones sexuales en los adolescentes), mientras que aquellos jóvenes con mayor nivel de preocupación y ansiedad, tienden a pensar que sus grupos de referencia sólo apoyarían la práctica de la abstinencia o retardo de las relaciones sexuales. De forma similar a la variable N, al hablar del nivel de Extraversión en los jóvenes los resultados sugieren que aquellos que tienden a ser sociables, de muchos amigos, arriesgados y en busca de excitación, se inclinan a pensar que sus grupos de referencia aprueban todas las conductas de protección, ya sea practicando la abstinencia o retardo, o mediante el uso de los métodos de protección. Coherentemente, se ha encontrado que la extraversión y el afecto positivo asociado a ésta, se relaciona con la confianza (Cortés, García, Monterrey, Fuentes y Pérez, 2000), lo cual podría explicar dicho resultado, en términos de la confianza que los jóvenes perciben que reciben por parte de sus grupos de referencia.

En cuanto a la norma subjetiva, la dinámica con las características de personalidad se dio de forma diferente a lo esperado de acuerdo con evidencias previas sobre esta característica de personalidad, ya que se esperaría que los jóvenes con altos niveles de preocupación y tendencia a presentar quejas somáticas, se mostraran altamente preocupados por cumplir las normas sociales sobre el manejo de su sexualidad y el uso de los métodos de protección; sin embargo, también se ha encontrado que un bajo afecto negativo se asociase con altos niveles de responsabilidad (López-Rosales y Moral-De la Rubia, 2001) por lo cual podría inferirse que mientras los jóvenes se muestren menos preocupados alrededor de alguna temática puntual, podrán desplegar de forma más efectiva conductas relacionadas con la responsabilidad sobre sus actos.

La relación negativa con la ansiedad social encontrada en algunos casos (Nieto-Andrade y Izazola-Licea, 1999), podría explicar por qué aquellos jóvenes con alto nivel de Psicoticismo presentan poca disposición para ceder ante la norma; al no enfocar su atención en la opinión de los grupos de referencia o la evaluación que éstos puedan hacer sobre él o ella, el joven no se interesa por sintonizar con las normas y expectativas externas y continúa comportándose de acuerdo con sus propios estándares.

Por su parte, la escala de Extraversión correlacionó de forma significativa y positiva con ambas sub escalas, pero únicamente en el grupo de jóvenes de 11 a 15 años. De manera que en este grupo, a medida que los jóvenes son más extrovertidos, presentan mayor disposición a complacer a sus grupos de referencia en la práctica de conductas sexuales saludables como el uso de los métodos de protección y la abstinencia o retardo de las relaciones sexuales.

Los resultados mencionados anteriormente relacionados con la responsabilidad y el afecto negativo, también ofrecen una explicación plausible a este resultado; la extraversión ha sido relacionada con el afecto positivo, el cual a su vez ha sido relacionado positivamente con la responsabilidad. Así, aquellos jóvenes con mayor nivel de extraversión tendrían una mayor tendencia a desarrollar conductas responsables, en este caso se sugiere que las conductas responsables son aquellas relacionadas con el cumplimiento de la norma percibida en el grupo de referencia. En este caso la escala de sinceridad no mostró correlación con ninguna de las dos sub escalas de norma subjetiva. Coherentemente con lo anterior, encontramos también una mayor responsabilidad de los jóvenes con características de extraversión y una menor preocupación de los jóvenes con características de dureza o Psicoticismo.

La correlación encontrada entre personalidad y control conductual percibido, se encuentra acorde con lo encontrado por otros autores (López, Vera y Orozco, 2001), quienes indican que la escala de Neuroticismo correlaciona negativamente con todas las medidas de autoeficacia, mientras que la de Extraversión correlaciona de forma positiva con dichas medidas (Romero, Luengo, Gómez y Sobral, 2002). La escala de Psicoticismo mantiene la tendencia observada anteriormente de tener una correlación significativa y negativa con las variables del TCP, presentándose menor confianza a medida que hay mayor nivel de Psicoticismo.

A pesar que en el estudio de Lasa, Bermúdez y Contreras (2002) se determinó que las variables de personalidad no juegan un papel determinante en la predicción de la 
intención de llevar a cabo una conducta, los resultados de la presente investigación indican algunas correlaciones significativas entre Neuroticismo e Intención de Riesgo y Protección (ambas negativas), resultan coherentes con los estudios de Romero et al. (Romero, et al., 2002) sobre la asociación de afecto negativo con una mayor capacidad del joven para protegerse. Adicionalmente, el nivel de la escala de Psicoticismo correlacionó negativamente con la Intención de Protección; se observa una correlación positiva (aunque no significativa) con la Intención de Riesgo, este dato parece sugerir que las características de dicha escala de personalidad podrían hacer a los jóvenes más vulnerables a involucrarse en conductas de riesgo.

La correlación significativa y positiva entre la escala de Sinceridad y la Intención de Riesgo de los jóvenes mayores y la Intención de Protección de los jóvenes menores; sugiere que en los adolescentes de 16 a 19 años, a mayor puntuación en la escala de Sinceridad (recordemos que sugiere ingenuidad en las relaciones sociales), presentan un mayor índice de riesgo; por su parte, en el grupo de los adolescentes entre 11 y 15 años, a mayor puntuación en la escala de Sinceridad se encuentra también mayores puntuaciones en la sub escala de índice de riesgo.

Sólo la Sinceridad correlaciona de forma consistente y significativa con el índice de comportamiento de riesgo, en relación directamente proporcional; se sugiere que aquellos jóvenes con mayor sinceridad y por tanto una mayor ingenuidad en las relaciones sociales (García-Izquierdo, García-Izquierdo y Ramos-Villagrasa, 2007; Perandones y Castejón, 2007), probablemente están más expuestos a los comportamientos de riesgo en comparación con los demás; esto se daría debido a que esta característica de personalidad les permite recibir reconocimiento y por tanto refuerzo social en sus interacciones, llevando a confiar erradamente en que los otros jóvenes tienen un interés no riesgoso, ignorando que pueden estar enfocados simplemente en una relación sexual.

Las escalas de Psicoticismo y Neuroticismo correlacionaron positivamente sólo en el grupo de jóvenes de 11 a 15 años; el Psicoticismo es directamente proporcional al índice de riesgo mientras que el Neuroticismo es inversamente proporcional al mismo. Estos resultados son coherentes con estudios anteriores donde se ha encontrado que altos niveles de Neuroticismo, bajos niveles de apertura y de amabilidad, se encuentran asociados con altos niveles de comportamiento de riesgo (García-Izquierdo, et al., 2007). Es decir que aquellos jóvenes con mayores niveles de ansiedad presentan mayor tendencia a involucrarse en comportamientos de riesgo mientras que los jóvenes con menos tendencia a relacionarse socialmente y con mayor retraimiento, se encuentran más alejados de los comportamientos riesgosos para su salud sexual y reproductiva.

La escala de Extraversión no mostró correlación con el comportamiento de riesgo, lo cual no es congruente con lo esperado de acuerdo con investigaciones previas; algunos estudios han evidenciado que la toma de decisiones impulsiva (característica de los jóvenes con altas puntuaciones en la escala de Extraversión) se constituye en un fuerte predictor de los comportamientos sexuales de riesgo (2002). Adicionalmente, la búsqueda de sensaciones (también característica relacionada con la escala E) correlacionó positivamente con las conductas desinhibidas y tendencia a involucrarse en conductas arriesgadas (2002). De forma específica, Schmitt (Eysenck y Eysenck, 1964) encontró que la Extraversión se asociaba directamente a conductas sexuales de riesgo como la promiscuidad. Es probable que los estilos de personalidad de la población de este estudio, caracterizada por altos niveles de sinceridad y estabilidad que ésta sugiere, más allá de la validez de la prueba, pudieran influir en la dinámica que se observa entre estas variables. De acuerdo con los anteriores resultados, se puede determinar que sí existe correlación entre las variables de la TCP y las características de personalidad en los jóvenes que participaron en la investigación.

A partir de los resultados discutidos, se formulan las siguientes conclusiones:

Existe correlación entre las características de personalidad y la actitud. La dirección de esta correlación depende principalmente de la escala de personalidad en cuestión; de forma consistente, se observó la tendencia a correlacionar de forma negativa el Neuroticismo y Psicoticismo y de forma positiva la Extraversión y la Sinceridad.

Existe correlación entre las características de personalidad y la norma subjetiva. La dirección de la correlación dependerá de las escalas de personalidad en cuestión, del aspecto de la norma subjetiva y de la conducta sobre la cual versa la norma subjetiva; así, se observa que en la percepción de aprobación y apoyo por parte de los grupos de referencia, a mayor Neuroticismo mayor fuerza de la norma subjetiva, mientras que la Extraversión correlaciona directamente sólo cuando se refieren al uso de los métodos de protección e inversamente cuando se trata de la abstinencia o retraso de las relaciones sexuales. El Psicoticismo correlaciona negativamente de forma consistente, mientras que la correlación de la sinceridad con esta variable no es concluyente.

En cuanto a la disposición a complacer al grupo de referencia, las correlaciones de Neuroticismo y Sinceridad son poco consistentes, mientras que la Extraversión correlaciona positivamente con dicha variable y el Psicoticismo correlaciona 
de forma negativa. Estas correlaciones se mantienen para el tercer factor de la norma subjetiva, mientras que la escala de Sinceridad presenta una correlación negativa levemente más consistente que en la variable anterior.

Existe correlación entre las características de personalidad y el control conductual percibido; el psicoticismo y la extraversión correlacionan de forma inversa y directa respectivamente, mientras que el neuroticismo y la sinceridad se inclinan más hacia las correlaciones negativas.

Existe correlación entre las características de personalidad y la intención. El neuroticismo guarda una correlación negativa al igual que el psicoticismo. Por su parte, extraversión y sinceridad guardan una correlación positiva.

Existe correlación entre las características de personalidad y los comportamientos de riesgo; el Neuroticismo guarda una correlación negativa, mientras que la sinceridad y el Psicoticismo presentan correlación positiva.

Los resultados presentan aportes relevantes para el ámbito clínico: las conclusiones previamente mencionadas, constituyen la evidencia sobre la cual un terapeuta podrá diseñar e implementar diversas estrategias específicas para la atención y prevención de problemáticas relacionadas con la salud sexual y reproductiva de la población adolescente. Es decir, las estrategias o técnicas a implementar podrían ser adaptables o intercambiables según las características de personalidad del paciente; adicionalmente, debemos tener en cuenta que en la población joven la personalidad se encuentra aún en consolidación, por lo tanto estos hallazgos podrían facilitar la orientación durante la adquisición comportamientos y prácticas más seguras en su sexualidad, previniendo así que se instauren comportamientos más estables que representen riegos en la sexualidad.

Resulta importante tener en cuenta para próximas investigaciones la inclusión de otras variables como los estilos de afrontamiento, los cuales podrían ofrecer un panorama mucho más amplio del comportamiento juvenil. Igualmente, se requiere abordar de forma más específica las características de personalidad, procurando una operacionalización más concisa de dichas características con miras al diseño de programas de intervención más precisos. Por último, es primordial fomentar investigaciones explicativas que incluyan las variables de la TCP en relación con la personalidad, ya que como se pudo observar, la mayoría de investigaciones se han centrado únicamente en el estudio del comportamiento de riesgo, ignorando constructos que guardan una relación dinámica con éste y pueden constituirse en piedra angular de programas de intervención.

\section{Referencias}

Ajzen, I. (2001). Nature and operation of attitudes. Annual Review of Psychology, 52, 27-58.

Ajzen, I. y Fishbein, M. (2005). The influence of attitudes on behavior. In D. Albarracín, B. Johnson y M. Zanna (Eds.), The handbook of attitudes (pp. 173-221 ). Mahwah, NJ: Erlbaum.

Baele, J., Dusseldorp, E., y Maes, S. (2001). Condom use self-efficacy: Effect on intended and actual condom use in adolescents. Journal of Adolescent Health, 28, 421-431.

Bello, A. y Palacio, J. (2012). Los comportamientos sexuales de riesgo en adolescentes del caribe a la luz de un modelo predictivo preliminar cognitivo social. Tesis de Doctorado en Psicología, Universidad del Norte, Barranquilla.

Bello-Villanueva, A.M., Palacio, J., Rodríguez-Díaz, M. y OviedoTrespalacios, O. (2013). Medición de la intención en la actividad sexual en adolescentes: Una aproximación de acuerdo al género del Caribe Colombiano. Terapia Psicologica, 31, 343-353. doi: http://dx.doi. org/10.4067/S0718-48082013000300009

Bello-Villanueva, A.M., Oviedo-Trespalacios, O., Vera-Villarroel, P., Oviedo, O., Rodriguez-Diaz, M., Celis-Atenas, K. y Pavez, P. (2014). Presentación de una escala para evaluar actitudes y creencias sobre la sexualidad reproductiva en adolescentes varones de la Región Caribe colombiana. Universitas Psychologica, 13, 47-60. doi:10.11144/Javeriana.UPSY13-1.peea.

Bello-Villanueva, A. M., Palacio, J., Vera-Villarroel, P., Oviedo-Trespalacios, O., Rodríguez-Díaz, M., Celis-Atenas, K. y Pávez, P. (2016) Construcción y Validación de una escala para evaluar salud sexual y reproductiva en adolescentes mujeres de la Región Caribe Colombiana. Universitas Psychologica. 15.

Carmack, C. C. y Lewis-Moss, R. K. (2009). Examining the theory of planned behavior applied to condom use: the effect-indicator vs. causal-indicator models. The Journal Primary Prevention, 30, 659-676.

Cortés, A., García, R., Monterrey, P., Fuentes, J. y Pérez, D. (2000). Sida, adolescencia y riesgos. Revista Cubana Medicinal General Integral, 16. Recuperado desde http://scielo.sld.cu/scielo.php?pid=S0864$21252000000300005 y$ script $=$ sci_arttext

Charnigo, R., Noar, S. M., Garnett, C., Crosby, R., Palmgreen, P., y Zimmerman, R. S. (2012). Sensation Seeking and Impulsivity: Combined Associations with Risky Sexual Behavior in a Large Sample of Young Adults. Journal of Sex Research.

Departamento Nacional de Planeación. (2012). Documento Conpes Social No. 147. Bogotá: Departamento nacional de Planeación.

DiClemente, R., Crittenden, C., Rose, E., Sales, J., Wingood, G., Crosby, R. y Salazar, L. (2008). Psychosocial predictors of HIV-Associated sexual behaviors and The Efficacy of Prevention Interventions in Adolescent at-Risk for HIV Infection: What works and what doesn't work? Psychosomatic Medicine, 70, 598-605.

Eysenck, H. J. (1970). Personality and attitudes to sex. A factorial study. Personality, 1, 355-370.

Eysenck, H. J. (1970a). A dimensional system of psychodiagnostics. In A. R. Mahrer (Ed.), New approaches to personality classification. New York: Columbia University Press.

Eysenck, H. J. (1970b). The structure of Human Personality. London: Methuen.

Eysenck, H. J. (1972). Personality and sexual behaviour. Journal of Psychosomatic Research, 16, 141-152.

Eysenck, H. J. (1973). Personality and attitudes to sex in criminals. The Journal of Sex Research, 9, 295-306.

Eysenck, H. J. y Eysenck, S. B. (1964). Manual of the Eysenck Personality Inventory. Londres: University of London Press.

Eysenck, H. J. y Eysenck, S. B. G. (2007). Cuestionario de Personalidad Para Niños (EPQ-J) y Adultos (EPQ-A). Madrid: TEA Ediciones.

Fishbein, M. y Ajzen, I. (1975). Belief, Attitude, Intention, and Behavior: An Introduction to Theory and Research. Reading: MA. 
Tschann, J. M., Flores, E., De Groat, C. L., Deardorff, J. y Wibbelsman, C. J. (2010). Condom negotiation strategies and actual condom use among Latino youth. Journal of Adolescent Health, 47, 254-262.

Fontanilla, S., Bello, A. y Palacio, J. (2011). Conocimientos, habilidades de aserción sexual y toma de decisiones en función de la intención de los comportamientos sexuales y reproductivos en adolescentes. Psicogente, 14, 294-309.

García-Izquierdo, A., García-Izquierdo, M. y Ramos-Villagrasa, P. (2007). Aportaciones de la inteligencia emocional y la autoeficacia: aplicaciones para la selección de personal. Anales de Psicología, 23, 231-239.

Hoyle, R. H., Fejfar, M. C. y Miller, J. D. (2000). Personality and sexual risk taking: A quantitative review. Journal of Personality, 1203-1231.

Inglés, C., Delgado, B., Bautista, R., Torregrosa, M., Espada, J., GarcíaFernández, J., . . García-López, L. (2006). Factores psicosociales relacionados con el consumo de alcohol y tabaco en adolescentes españoles. International Journal of Clinical and Health Psychology, 7, 403-420.

Jemmott, J. B., Heeren, G., Ngwane, Z., Hewitt, N., L., J., Shell, R. y O'Leary, A. (2007). Theory of planned behaviour predictors of intention to use condoms among Xhosa adolescents in South Africa. AIDS Care, 19, 677-684

Kalichman, S. C., Cain, D., Zweben, A. y Swain, G. (2003). Sensation seeking, alcohol use and sexual risk behaviors among men receiving services at a clinic for sexually transmitted infections. Journal of Studies on Alcohol and Drugs, 64, 564-569.

Kalichman, S. C., Simbayi, L., Jooste, S., Vermaak, R. y Cain, D. (2008). Sensation seeking and alcohol use predict HIV transmission risks: prospective study of sexually transmitted infection clinic patients, Cape Town, South Africa. Addictive Behaviors, 33, 1630-1633.

Kalichman, S. C., Simbayi, L. C., Jooste, S., Cain, D. y Cherry, C. (2006). Sensation seeking, alcohol use, and sexual behaviors among sexually transmitted infection clinic patients in Cape Town, South Africa. Psychol Addict Behaviors, 20, 298-304.

Lasa, A., Bermúdez, J. y Contrera, A. (2002). Personalidad, procesos psicológicos e intención de cambio de conducta implicaciones para el desarrollo de conducta saludable. Acción psicológica, 1, 151-164.

López-Rosales, F. y Moral-De la Rubia, J. (2001). Validación de una escala de autoeficacia para la prevención del SIDA en adolescentes. Salud Pública México, 43, 421-432.

López, N., Vera, L. y Orozco, L. (2001). Diferencias en los conocimientos, actitudes y comportamientos sexuales relacionados con el SIDA, entre hombres y mujeres jóvenes de Bucaramanga. Colombia Médica, $32,32-40$.

Llewellyn, D. J. (2008). The psychology of risk taking: toward the integration of psychometric and neuropsychological paradigms. American Journal Psycholy, 121, 363-376.

Martín, M. J., Martínez, J. M. y Rojas, D. (2011). Teoría del comportamiento planificado y conducta sexual de riesgo en hombres homosexuales. Revista Panamerica de Salud Publica, 29, 433-443.

Ministerio de Salud y Protección Social (2013) Boletín epidemiológico, situación del VIH/Sida Colombia 2013. Recuperado desde https://www. minsalud.gov.co/Documentos\%20y\%20Publicaciones/BOLETIN\%20 EPIDEMIOLOGICO\%20VIH\%201983-2012.pdf

Nieto-Andrade, B. y Izazola-Licea, J. (1999). Uso del condón en hombres con parejas no estables en la Ciudad de México. Salud Pública México, 41, 85-94.

Ntaganira, J., Hass, L. J., Hosner, S., Brown, L. y Mock, N. B. (2012). Sexual risk behaviors among youth heads of household in Gikongoro, south province of Rwanda. BMC Public Health, 22, 225.

Papalia, D., Wendkos, S. y Duskin, R. (2009). Psicología del desarrollo. México: McGraw Hill.

Perandones, T. y Castejón, J. (2007). Estudio correlacional entre personalidad, inteligencia emocional y autoeficacia en profesorado de Educación Secundaria y Bachillerato. Paper presented at the V congreso internacional "educación y sociedad".

Profamilia. (2010). Encuesta Nacional de Demografía y Salud. Bogotá: Asociación Probienestar de la Familia Colombiana. Bogotá, Colombia.
Romero, E., Luengo, M. A., Gómez, J. A. y Sobral, J. (2002). La estructura de los rasgos de personalidad en adolescentes: el modelo de cinco factores y los cinco alternativos. Psicothema, 14, 134-143.

Sierra, R. C., Oviedo-Trespalacios, O., Candelo, J. E. y Soto, J. D. (2015). The influence of atmospheric conditions on the leakage current of ceramic insulators on the Colombian Caribbean coast. Environmental Science and Pollution Research, 22, 2526-2536.

Vargas-Trujillo, E., Barrera, F., Burgos, M. C. y Daza, B. C. (2006). La intención de los jóvenes de tener relaciones sexuales en la adolescencia: el papel de la televisión, la relación con los padres y las cogniciones. Universitas psychologica, 5, 69-84.

Zuckerman, M. y Kuhlman, D. M. (2000). Personality and risk-taking: common biosocial factors. Journal of Personality, 68, 999-1029. 
